\title{
Design and Analysis of All-Terrain Electric Vehicle
}

\author{
Gowtham Rajan $\mathrm{N}^{(1)}$, Deepak B Prasad ${ }^{(2)}$, \\ Pre-Final $^{(1,2)}$ Year Students, \\ Department Of Mechanical Engineering, \\ Hindusthan College Of Engineering And Technology, \\ Coimbatore 641 032, India.
}

\author{
Anwar Wasim A ${ }^{(3)}$, Gowtham $\mathrm{K}^{(4)}$, Ajith George ${ }^{(5)}$, \\ Pre-Final $^{(3,4)}$ and Second ${ }^{(5)}$ Year Students, \\ Department Of Mechanical Engineering, \\ Hindusthan College Of Engineering And Technology, \\ Coimbatore 641 032, India
}

\begin{abstract}
Science is a double-edged tool of the modern world which promoted revolution in a transportation industry. Utilization of coal, oil, diesel exudes a lot of debased gasses, this outcomes in less than ideal equality of ozone layer improvement, Global warming and climatic changes, etc. As well oil usage on the planet increases, similarly as oil creation decays. So now the world is trading their transportation sector absolutely reliant on electrical and electronic as a power source. This foreseen the need to contribute a development that supports green energy, consider this condition we could improve and utilize electricity to charge electric vehicles rather than using conventional engines that are executed in the vehicle. This exploration paper covers the design and examination of the roll cage, suspension framework, steering framework, braking mechanism and power train arrangement of an All-Terrain Electric Vehicle. The vehicle is intended to explore intense landscapes in which the ordinary vehicles can't perform. It is likewise structured remembering the commercial suitability. The design tries to advance the current model and advance it with an improved framework. The vehicle has been made keeping, as a main priority the different structure viewpoint, which have been expressly indicated in the automobile safety guidelines.
\end{abstract}

Keywords-Buggy Vehicle, Electric Terrain vehicle, Off road, Single Seater, Ebaja Vehicle.

\section{INTRODUCTION}

All-terrain vehicles are one the most famous vehicles and which were first developed in the US in the early 70's and Since then the use of these vehicles has increased. An Allterrain electric vehicle can be used for on-road as well as offroad manoeuvrability and applications like farms, valley rides, hill climbing etc. The prominent design features customized steering knuckles, cluster board, adept roll over protection, driver ergonomics and modular components. The design of the vehicle followed the various

requirements of the people. The designer should meet the consequences of the problems that are put forth by the customers. Following are the major points which were considered for ensuring a complete off-road vehicle. Few mandatory requirements of customers are list below: Endurance, safety and ergonomics, Market availability, Cost the components, Maneuverability, Easy handling. We begin the task of designing and conducting extensive research on major parts of the vehicle. However, the design was kept modifying the basis of our requirements.

\section{DESIGN}

The design and development process of the vehicle components involves various factors namely material selection, frame design, cross-section determination and finite element analysis. But the foremost purpose of the design is to achieve a weight reduction compared to the existing other terrain design and achieve optimum off-road performance without compromising on the driver safety, reliability and durability. To ensure that the optimal material is chosen, extensive research was carried out and compared with materials from multiple categories. The key categories for comparison were strength, weight to strength ratio and cost [6]. The details for each step are given below

\section{A. Vehicle concept:}

This vehicle is a first-generation prototype. During the brainstorming stage, several design ideas were considered and the optimum solution at every stage was pursued, Various design and analysis software's such as Solidworks, Adams, Ansys, and Solidworks Electrical were employed to help bring the vision to reality into an easily usable digital medium that is suitable for design creation, analysis and optimization.

Based on last year's design we identified certain aspects which could be optimized, and these topics dominated our early discussions. For instance, since we had to reduce the weight of the vehicles drastically, we decided to change the piping dimensions of the vehicles for both primary and secondary.

We were also discussing as to whether it was better to use the trailing arm suspension or go with the conventional double wishbone system. We ultimately decided that we would use the double wishbone system as the higher variable load 
bearing double wishbone systems benefits outweighed the light weight trailing arm suspension system. Since the vehicle had to be operated in rough conditions, the double wishbone system was deemed necessary.

We also brainstormed and came to the conclusion to use an Ackerman steering system as an understeer since we operated at relatively low speeds and the slip angle was reduced. This gives greater maneuverability to the driver. CAD model is shown in Fig. 1.

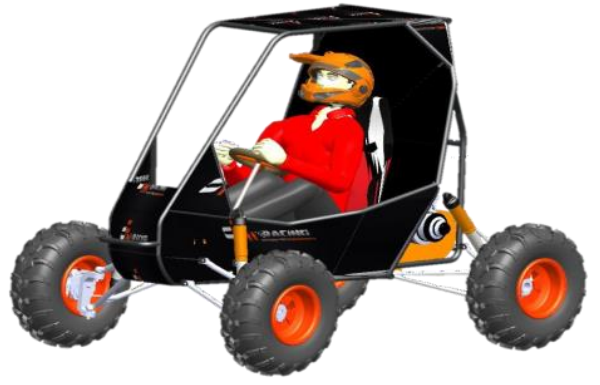

Fig. 1 CAD Model

\section{B. Frame:}

The chosen design is a tubular space frame composed of a combination of two sets of tube members. The primary members are made entirely of 1.25 " O.D and $1.65 \mathrm{~mm}$ thickness, whereas the secondary members are made up of 1 " O.D and $1.65 \mathrm{~mm}$ thickness. These tubing were carefully chosen keeping in mind that it had to satisfy the bending strength requirements. The decision for choosing two sets of tube members was motivated by the fact that it results in significant weight reduction compared to the previous year's design which employed the 1" O. D for both primary and secondary members at thickness of $3 \mathrm{~mm}$ and $1 \mathrm{~mm}$. The tubing varies across the chassis depending upon the severity and various possible failure modes.

After finalizing the frame along with its material and cross section, it is very essential to test the validity, strength and deformation of the frame in its worst-case scenario. The frame should withstand the impact, roll over conditions and provide at most safety to the driver. Following tests were performed on the roll cage.

\section{Material Selection:}

The material for the members was chosen as AISI 4130 (Chromoly) Steel since it offers high tensile strength, excellent ductility, high impact strength, corrosion resistance and good weldability at a reasonable price and availability. Even though it may not be lighter than other steels, it is stronger and hence we can use less. To improve its strength, it was normalized before its usage.

D. Chassis design:

A nose design was adopted in order to enhance driver ergonomics. This design reduces blind spots in the front position of the car for the driver and increases his field of view. In the previous year a non-nose design was used. But in order to make the roll cage more compact, the nose design was implemented. When compared to the non-nose design, the nose design has very fewer individual members making the nose design easier to manufacture as it will require the least cutting and welding of individual tubes. Discrete view section of design is shown in Fig. 2 Fig. 3 Fig. 4 Fig. 5

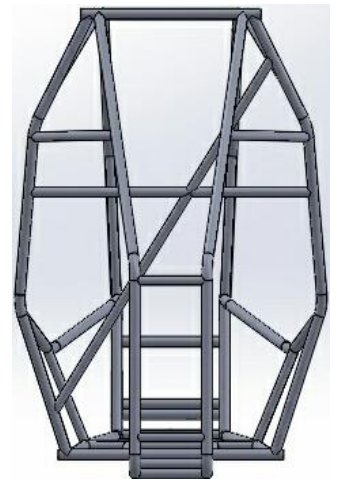

Fig. 2 Front View

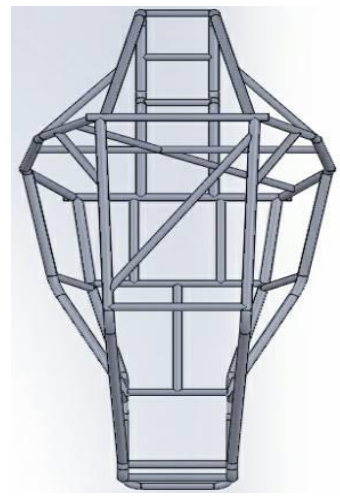

Fig. 4 Top View

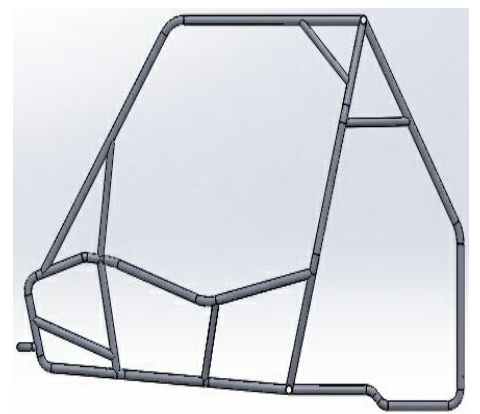

Fig. 3 Side View

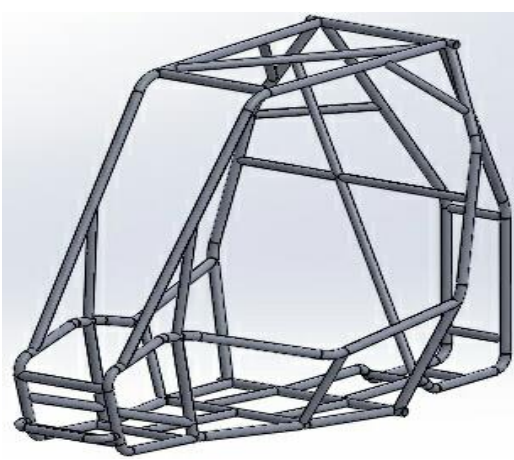

Fig. 5 Isometric View

\section{E. Testing:}

Designs have been primarily validated through extensive FEA analysis. Due to the rigorous academic schedule, testing opportunities have been limited. However, the important tests such as material tests, impact test, weld strength test, brake performance test and suspension test were thoroughly carried out using a combination of nondestructive testing methods and FEA analyses. The results of testing were carefully scrutinized to ensure that reliability of performance can be established.

\section{CAE ANALYSIS}

The CAE software that was used for analysis was Ansys. The three-dimensional geometry which was developed in SolidWorks was imported into Ansys and various analysis like Front impact analysis, rollover analysis, and wheel bump were performed.

All these analyses were based on the conditions that we would experience in the Baja track and remote loads were given accordingly. The hard points were fixed and the rest of the loads were assigned according to the G-Force calculations.

\section{A. Front impact}

The worst case for the front impact of ATV.is assumed to hit a vehicle at velocity of $16.66 \mathrm{~m} / \mathrm{s}$. This will give a maximum 
possible work done. The impact force was calculated using the kinetic energy transfer.

Work done $=$ Change in kinetic Energy

$$
=\left(0.5 \mathrm{mv}^{\wedge} \text { 2initial }-0.5 \mathrm{mv}^{\wedge} 2 \text { final }\right)
$$$$
=0.5(240 * 16.6672)
$$

Work done $=33,334.6 \mathrm{Nm}$

Now,

Work done $=$ force $*$ Displacement

Displacement $=$ impact time* ${ }^{*} \max$

Impact time $=$ Weight of the vehicle $*($ Velocity/load $)$

impact time $=0.119 \mathrm{sec}$

Displacement $=0.119 * 16.667$

$$
=2 \mathrm{~m}
$$

Force $=$ Work done $/$ displacement

$$
=33,334.6 / 2
$$

Force Due to front impact $=16,664 \mathrm{~N}$

By analyzing,

Stress factor of safety $=2.16$

Maximum stress produced $=201.25 \mathrm{Mpa}$

Maximum total deformation $=0.188 \mathrm{~mm}$

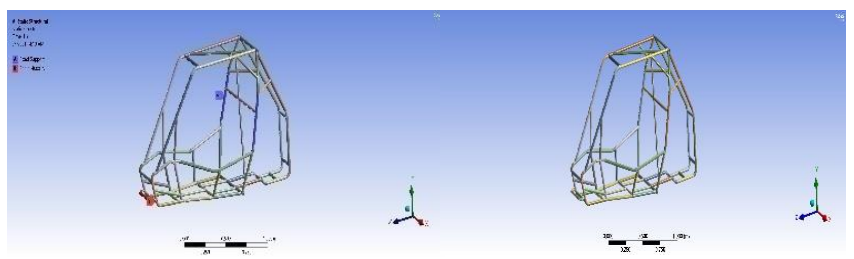

Fig. 6 Boundary Condition

Fig. 7 Factor of Safety

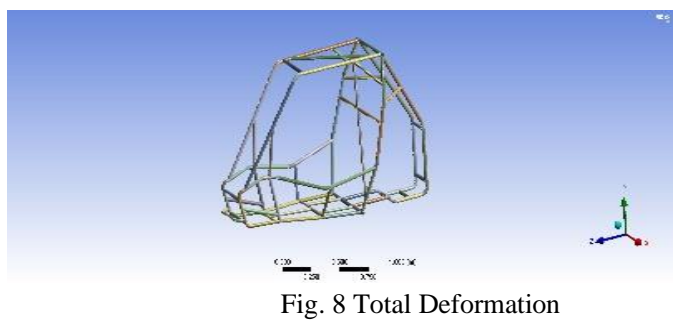

\section{B. Rear impact}

In rear collision, the ATV is assumed to be stationary, fixed and another ATV with Mass of $240 \mathrm{~kg}$ with velocity of 16.66 $\mathrm{m} / \mathrm{s}$ collides with the former ATV which in stationary Force is applied on rear portion of ATV and all DOF'S of front were constrained. The ATV in motion transfers all of its kinetic energy to the fixed ATV and comes to rest. Thus, the forces calculated for frontal impact will be the same for rear impact test. i.e. $16,664 \mathrm{~N}$

By analyzing,

Stress factor of safety $=2.153$.

Maximum stress produced $=202.04 \mathrm{Mpa}$

Maximum total deformation $=1.891 \mathrm{~mm}$

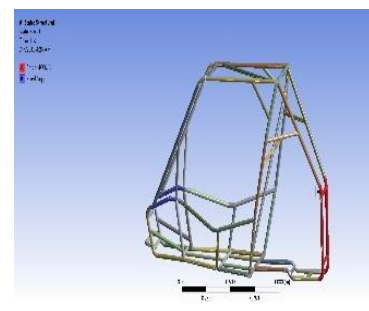

Fig. 9 Boundary Condition

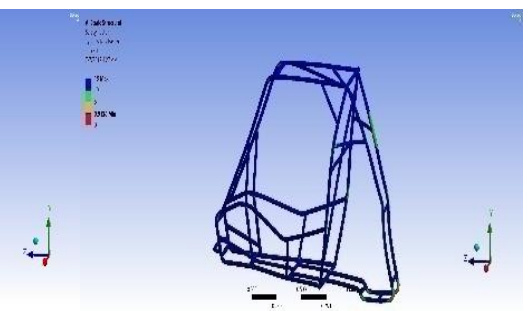

Fig. 10 Factor of Safety

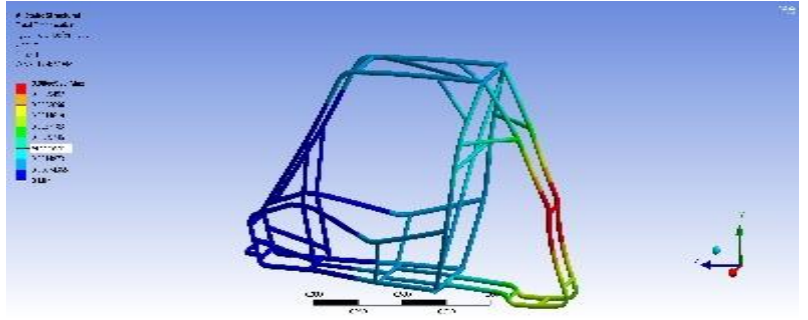

Fig. 11 Total Deformation

C. side impact

During side impact analysis another ATV will allowed to hit the buggy on side deformable body. For analysis, ATV is considered to be in static state and force corresponding to the velocity $16.66 \mathrm{~m} / \mathrm{s}$ with equal mass of $240 \mathrm{~kg}$ is applied to side of the roll cage of ATV keeping suspension members fixed.

By analyzing,

Stress factor of safety $=1.787$.

Maximum stress produced $=202.04 \mathrm{Mpa}$

Maximum total deformation $=1.376 \mathrm{~mm}$

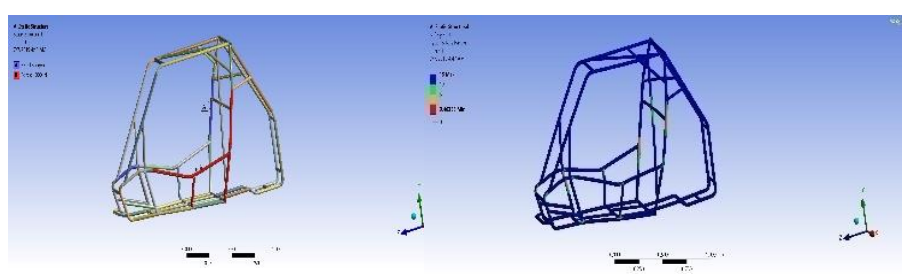

Fig. 12 Boundary Condition Fig. 13 Factor of Safety

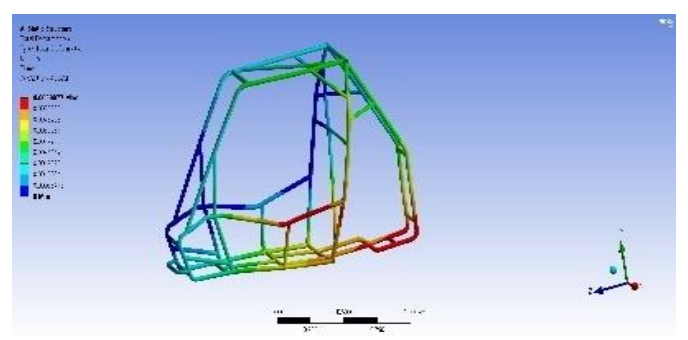

Fig. 14 Total Deformation

\section{Roll over}

In roll over condition, while turning the vehicle at higher velocities there occurs the cornering force, which is nothing but the centrifugal force acts on the tire which results in the rollover of the vehicle. Therefore, the force involved here was calculated by

Centrifugal force $=\left(\mathrm{m}^{*} \mathrm{v}^{\wedge} 2\right) / \mathrm{r}$

Where,

$\mathrm{m}=$ mass of the vehicle

$\mathrm{v}=$ velocity of the vehicle

$\mathrm{r}=$ Turning radius

Centrifugal force $=(240 * 16.67) / 2.7$

$$
=1097282 \mathrm{~N}
$$

By analyzing,

Stress factor of safety $=2.42$

Maximum stress produced $=179.6 \mathrm{Mpa}$

Maximum total deformation $=1.014 \mathrm{~mm}$ 


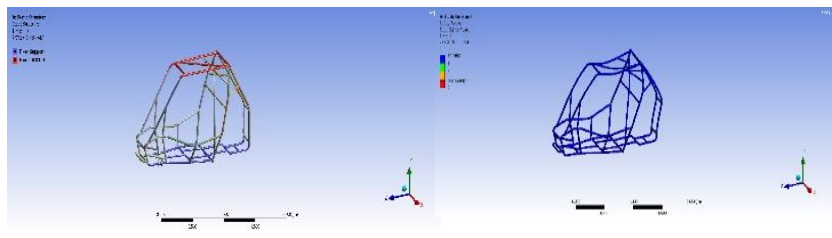

Fig. 15 Boundary Condition

Fig. 16 Factor of Safety

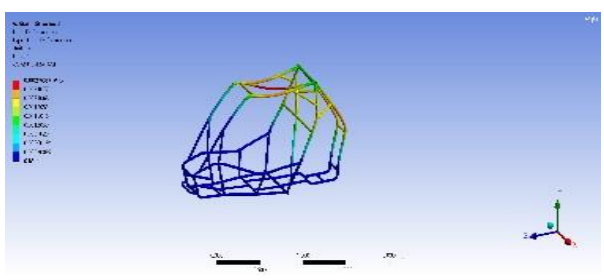

Fig. 17 Total Deformation

\section{SUSPENSION}

While designing the suspension system, various options such as MacPherson struts, trailing arms and live axle were considered.

Initially there was an inclination on using trailing arm during initial discussions but decided against it as we came to the conclusion that it would not be able to withstand the variable heavy loads. Eventually, a decision was made in favor of an independent double wishbone suspension system. This is mainly due to the system's proven success in off-road applications along with its flexibility with regard to wheel movement and critical suspension geometry characteristics. The suspension system allows for complete camber and toe adjustment both in the front and rear of the vehicle. The various suspension parameters like wheel jounce, rebound, camber gain, motion ratio, etc. were thoroughly analyzed using ADAMS software. Fig. 18 shows the CG stimulation of vehicle [1].

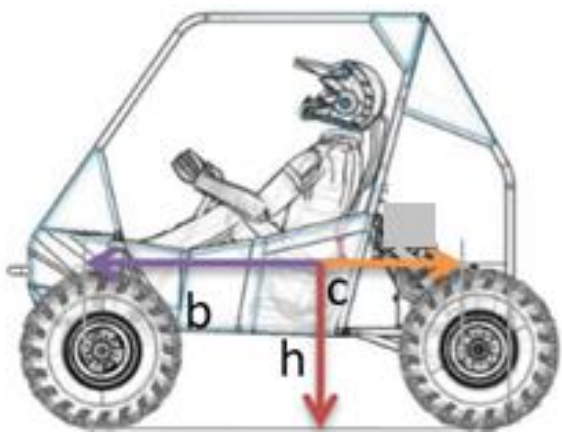

Fig. 18 CG stimulation

\section{A. A-Arms}

All the A-Arms were fabricated from 4130 secondary steel tubing by a combination of notching and welding. The mounting points were fixed at the design stage using SolidWorks software. The upper front and rear A Arm mounts were specially laser cut in order to reduce weight by avoiding extra members for mounting. The designs for the lower and upper A-Arms at the front and rear were comprehensively evaluated under a variety of loading conditions. This was done to ensure that they hold up even under the most extreme driving conditions.

\section{B. Shocks}

The shock absorbers used in the vehicle are FOX Racing Air Shocks. These shocks were preferred for their bump and rebound damping adjustment at low as well as high speeds. The shocks also feature a wide range of spring rates. Due to the uneven distribution of weights between the front and the rear portions of the vehicle, the stiffness of the rear shock absorbers has been kept higher than that of the front. Both sets of shocks have a maximum travel of 8.5 ". This facilitates better maneuverability under all driving conditions.
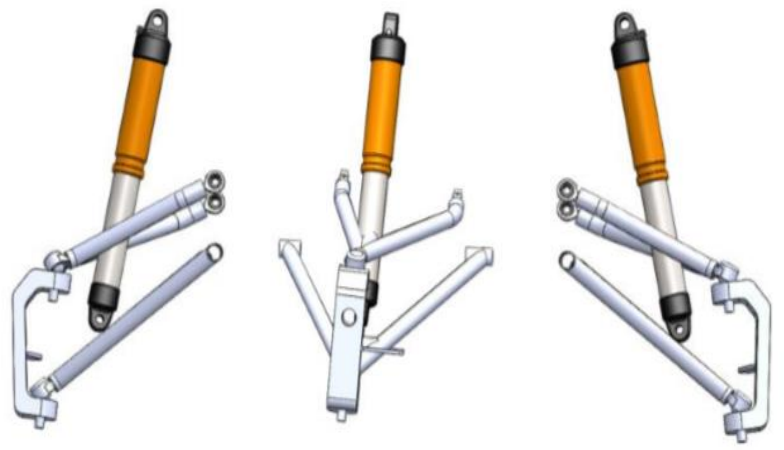

Fig. 19 Shock Absorber mount with discrete views

\section{Uprights}

A lot of thought was put in for the usage of material for the uprights. There were plenty of discussions whether to use cast iron or aluminum. Aluminum is a ductile material and has very high shear strength. It is light weight and strong. Compared to cast iron it can be easily machined. The wastage while machining is also very less in aluminum. Since cost was not going to be a problem this time around since we covered the expenses and we decided to opt for aluminum. Taking into consideration parameters like steering geometry, suspension geometry, heat dissipation, dynamic and static stress acting on the uprights we designed the uprights in SolidWorks and analyzed the forces acting on it in Ansys. We used Al 7075 for the uprights and based on our ground work we felt the 7 series would be more apt than the 6 series. We used in house fabrication and machined an aluminum block using CNC.

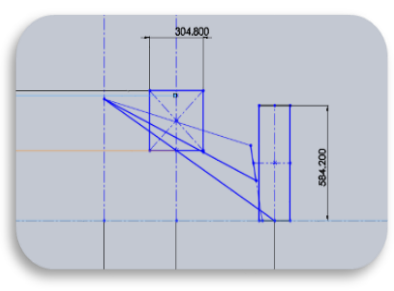

Fig. 20 Analytical Method

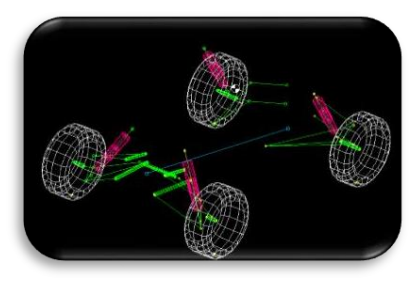

Fig. 21 Stimulation Method

\section{WISHBONE:}

Double Wishbone Suspension System consists of two lateral control arms (upper arm and lower arm) usually of unequal length along with a coil over spring and shock absorber. It is popular as front suspension mostly used in rear wheel drive vehicles. Design of the geometry of double 
wishbone suspension system along with design of spring plays a very important role in maintaining the stability of the vehicle. This type of suspension system provides increasing negative camber gain all the way to full jounce travel unlike Macpherson Strut. They also enable easy adjustment of wheel parameter such as camber. Double wishbone suspension system has got superior dynamic characters as well as load handling capabilities.

\section{E. ANALYSIS OF WISHBONES:}

Analysis of wishbone in Ansys Analysis Software is necessary in order to determine the induced maximum stress and maximum deflection in wishbones, for analysis, wishbones are first needed to be modelled in software. The modelling of wishbones is done in SOLIDWORKS modelling software. It is engineering simulation software (computer-aided engineering). Various types of analysis like structural analysis, thermal analysis, etc., are possible using ANSYS analysis software. In structural analysis in ANSYS, boundary conditions are to be defined in order to determine the stress and deflection. After modelling the wishbones in Solid works modelling software, these models were imported into Ansys Analysis Software. Various boundary conditions and load cases were applied for determining the maximum stress and maximum deflection for wishbone.

Bump force $=$ wheel rate $*$ distance travel by wheel

$$
\begin{aligned}
& =25,714 * 8 \\
& =5225.08 \mathrm{~N}
\end{aligned}
$$

Maximum damping load $=3728 \mathrm{~N}$

Therefore,

Bump force acts on the knuckle

$$
=1496 \mathrm{~N}
$$

By analyzing,

Stress factor of safety $=4.95$

Maximum stress produced $=87.765 \mathrm{Mpa}$

Maximum total deformation $=0.115 \mathrm{~mm}$

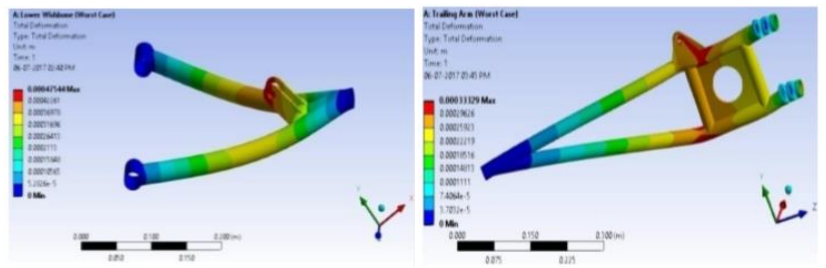

Fig. 22 Analyzed A-Arm and Trailing arm

\section{F. $H u b$}

In automotive suspension, a steering upright is that part which contains the wheel hub or spindle, and attaches to the suspension components, variously it is known as steering knuckle, spindle, upright or hub. The wheel and tire assembly attach to the hub or spindle of the knuckle where the tire/wheel rotates while being held in a stable plane of motion by the knuckle/suspension assembly. The double-wishbone suspension, the knuckle is attached to the upper control arm at the top and the lower control arm at the bottom. The wheel assembly is shown attached to the knuckle at its center point. Suspension systems in any vehicles uses different types of links, arms, and joint to let the wheels move freely, front suspensions also have to allow the front wheel to turn. Steering knuckle/spindle assembly, which have two separate or one complete part attached together in one of these links. Hub is the part attached to upright, the purpose of a wheel hub is to attach a wheel to a motor shaft. Hub are also used to attach lifting arms, release doors and pulleys to motor shafts Wheels are typically attached to hubs via the wheels face or its center. The wheel is attached through fasteners to hub due to a good strength and can be easily removed for storage or servicing. Hubs are typically attached to the motors by closely sliding over and locking into engagement with their shafts transferring torque from the motor, through the hub and to the wheel. Hub must be capable of rigidly Supporting its share of the total weight of a vehicle without failure during its expected life span. If the hub geometry and material selection are inadequate, it will brake assembly which cannot be repaired. The hub is made of aluminum 6 series and is very easily machinable and is designed in a way to withstand all possible loading conditions. It was analyzed in Ansys where the bolted points were fixed and hoop's stress acted on it.

\section{STEERING}

The design of the steering system was carried out prioritizing reduction in steering effort and improved road response. The various options that were considered for the steering system were rack and pinion mechanism, five-linkage mechanism, hydraulic steering and an automated electrical system Among these, the rack and pinion mechanism were preferred because of its simplicity, compact size, and ease of packing in the vehicle's toe-box and its proven track record of success in off-road applications. The steering system follows Ackermann steering principle with an Ackermann percentage of $96 \%$. The tie rod length and the roll center were determined by kinematic analysis [4]

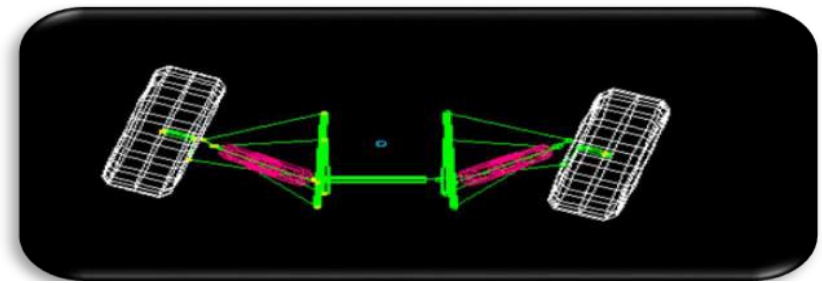

Fig. 23 Lotus Stimulation

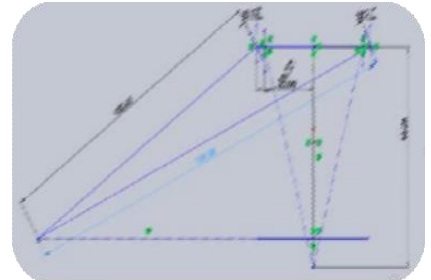

Fig. 24 Analytical Method

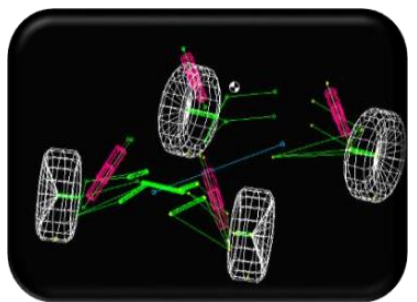

Fig. 25 Stimulation Method
Steering Type Rack and pinion Steering Geometry Ackerman Geometry Ackermann percentage 96\% Steer Type Understeer Steering Column Type Tilting Type of Drive Centre Camber Change 4.5 degrees over the travel Static Castor Angle 6 degrees King Pin Inclination 5.8 degrees Toe in (Front) 0 
degrees Scrub Radius 60mm Turning Radius $2.5 \mathrm{~m}$ to right Effective Rack Length $355 \mathrm{~mm}$ Power Assist No. [2]

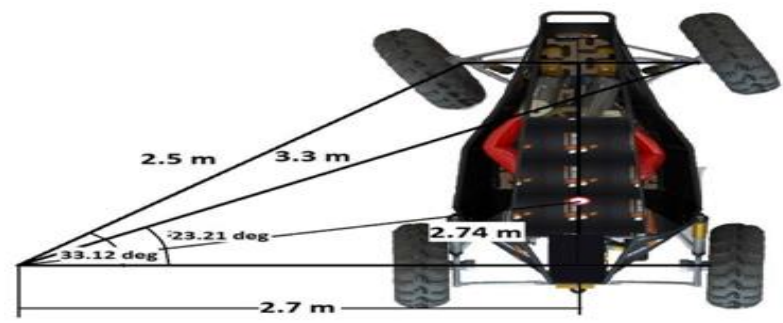

Fig. 26 Steering Geometry

\section{BRAKES}

While designing the brake system for the vehicle, the team considered two major systems. One system consisted of using a single tandem master cylinder with two cylinders and pistons with a Pressure Regulating Valve (PRV) positioned across the hydraulic lines. The second system consisted of using a dual master cylinder arrangement with two separate master cylinders, each forming a separate hydraulic circuit. A bias bar or balance bar performs the role of a PRV in this system, mechanically dividing the brake force across each master cylinder depending on pre-determined bias requirements. Of the two options, the second option was preferred. This is because, compared to a tandem master cylinder, a dual master cylinder arrangement reduces driver effort at the pedal, thereby minimizing driver fatigue. Also, the system offers an inherent safety interlock, wherein even if one cylinder (one hydraulic circuit) fails, the other cylinder remains in an operable condition.

Another disadvantage of a tandem master cylinder is its requirement of a PRV to create the necessary braking bias. A PRV is positioned along the hydraulic lines. While adjusting a PRV, it is very possible that air may enter the hydraulic circuits, diminishing braking performance, or under extreme circumstances, it may even compromise the braking system.

In a dual master cylinder arrangement, the brake bias can be adjusted mechanically using a bias bar or a balance bar. Since the tires have positive scrub radius, a horizontal-split type brake circuit has been preferred. Hence, one master cylinder will control the front braking circuit and the other cylinder will control the rear braking circuit.

\section{A. Master Cylinders}

The bore size of the cylinders has to be as small as possible in order to provide large hydraulic pressure in the circuit at minimal brake effort. At the same time, the cylinder must also be able to withstand the applied pressure. To achieve a balance between the two factors, two Wildwood aluminum master cylinders with $5 / 8$ inch bore diameter were preferred.

\section{B. Calipers}

While selecting the type of caliper to be used, there were two major considerations for the team. One option was to use a fixed-type caliper with cylinders and pistons on both sides of the brake pads. Such a caliper would offer strong braking performance, but at the expense of increased driver effort and increased unsprang mass of the vehicle. The use of such calipers is usually not recommended without brake boosters and are generally used only with heavy vehicles such as trucks.

The other option was to use a floating-type caliper, which has pistons and cylinders on only one side and uses a sliding member to push the brake pads on the other side. Such calipers have the advantage of offering increased pad contact area and more uniform pad wear. They are also compact, light-weight and suitable for packaging. In addition to that, they also have fewer leak points compared to fixed-type calipers.

The obvious advantages of floating-type calipers convinced the team to use them on all four wheels. In order to have a larger contact area for the brake pads, dual piston floatingtype calipers of 1 " bore diameter were selected. The calipers were mounted such that the bleeding valve always faces upwards.

\section{Brake Disc}

The diameter of a brake disc greatly influences the braking torque. Larger the brake disc diameter, larger the effective braking radius and hence, larger the braking torque. For Vehicle E165, the brake discs on all four wheels have a diameter of $180 \mathrm{~mm}$ and a thickness of $6 \mathrm{~mm}$. The discs are made from mild steel by CNC machining.

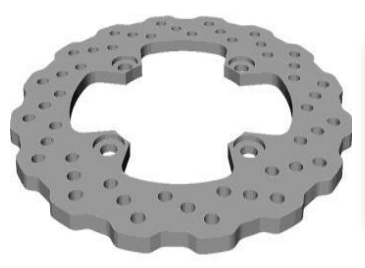

Fig. 28 CAD Drawing

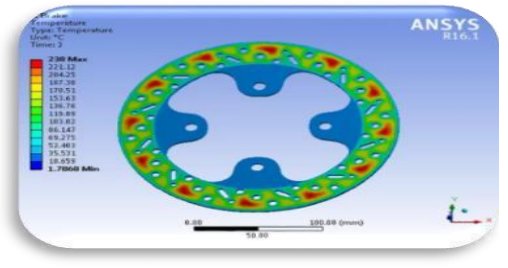

Fig. 29 Analyzes Result

\section{Balance Bar}

A balance bar is a threaded rod, usually an adjustable lever, which pivots on a spherical bearing located at its center. The bar is supported by clevises at either end. Master cylinder push rods are threaded into the clevises in a direction perpendicular to the balance bar.

When the balance bar is centered, it pushes equally on both master cylinders creating equal pressure, given that the master cylinders have the same size bore. When adjusted as far as possible towards one master cylinder it will push approximately twice as hard on that cylinder as the other.

The use of the balance bar enables easy adjustment of brake bias in a mechanical way without interfering with the hydraulic lines. The entire pedal assembly including the brake pedal, balance bar, clevises and base plate for mounting the master cylinders were fabricated in-house by the team members. 


\section{POWERTRAIN AND ELECTRICALS}

The team has used the e-kit components provided by Compage Automation Systems. These components include a lithium battery pack consisting of four packs of cell each of 2.6 A-h / 3.7 V, a DC motor, a controller, a main contractor, a low voltage and a high voltage harness, an F-N-R switch, an accelerated pedal, a brake switch, a universal coupling, a charging unit and a single stage reduction gearbox with a reduction ratio of 12:1. The gearbox can be directly mounted with the motor. A CV joint is provided at the output of the gearbox integrated with it.

\section{VIII.OPERATIONS INVOLVED IN VEHICLE}

The batteries are from the outset totally energized and a while later they are related with a motor for releasing. This helps with keeping the battery discharged reliably. This is moreover done, as the efficiency of the Brushless DC motor is around $85-90 \%$. Subsequently, under this condition the battery gets totally deenergized. From the start the supply is given through the controller to the motor it takes a high starting current to push the wheel to move forward. The vehicle is around $180 \mathrm{~kg}$ barring the driver, it can reach up to $40 \mathrm{~km} / \mathrm{h}$ speed at the best. The batteries get released reliably, consequently it gives the unending race to the vehicle. Vehicle is absolutely depending upon the driver's capacity on the choke pedal to move speed. As the speed varies the store current similarly vacillates. In this way, the speed assortment must be low to keep the battery alive for the most extraordinary term of time. For ending the vehicle, brake pedals were given in the vehicle; from that point on the circle brakes should be applied. The circle brakes can be applied in a brief moment during crisis anyway this should be kept up a vital good way as this could hurt the motor and besides produce futile back emf. The typical battery back-up is around keep going for more than two and half hours. An optional battery is utilized in the vehicle to control an assistant electrical gadget like voltmeter, ammeter thus for.

\section{CONCLUSION}

The vehicles were manufactured by the design and amassed. The vehicle execution was then approved by performing different tests. Estimation of the static boundaries, for example, track width, stature, length was completed and was seen as inside the cutoff points indicated. The dynamic testing of the vehicle was completed on numerous occasions and was seen as acceptable in the parts of driver security, driver comfort, simplicity of directing and maneuverability end, the execution of the plan in the vehicle accomplished the objectives of ideal execution by adaptability of camber alteration, least toeing, driver solace and most extreme travel; these were accomplished at a sensible expense.

\section{ACKNOWLEDGEMENT}

This paper is a complete researched, real time project designed and fabricated by the students of Hindusthan College of Engineering and Technology, Coimbatore. The research, vehicle design, analysis, fabrication and testing was made possible with the help and assistance provided by Dr. Y. Ras Mathew, Managing Director of Hi-Racing motor sports, and Associate professor in Hindusthan College of Engineering and Technology, Coimbatore. The project was taken by a team of 10 members namely Chandra kanth, Deepak B Prasad, Anwar Wasim A, Ajith George, Gowtham K, Gowtham Rajan N, Nithish K C, Raj Kumar, Parthasarathi, Sri Balaji.

\section{REFERENCES}

[1] Akshay G Bharadwaj, Sujay M , Lohith E, Karthik S Design, Analysis, Simulation and Validation of Suspension System for an Electric All-Terrain Vehicle (ATV) "International Journal of Innovative Research in Science, Engineering and Technology"Vol. 5, Issue 12, December 2016.

[2] Jukanti Sandeep Reddy, S Sai Dheeraj, S Upendar Reddy, Design and Structural Analysis of Steering Knuckle for An Electric All-Terrain Vehicle - E Baja, "International Journal Of Engineering Research \& Technology" Volume 08, Issue 08 (August 2019).

[3] Pushkar.B. Suryavanshil A.D. Desai, Designing continuously Variable Transmission for All-Terrain-Vehicle - A Review "International Journal for Scientific Research \& Development" Vol. 4, Issue 05, 2016

[4] Jukanti Sandeep Reddy, S Sai Dheeraj, S Upendar Reddy, Design and Structural Analysis of Steering Knuckle for An Electric All-Terrain Vehicle - E Baja, "International Journal of Engineering Research \& Technology", Vol. 8 Issue 08, August-2019.

[5] Da Wen Ge, SugengAriyono and Daw ThetThet Mon "A review on continuously variable transmissions control," National Conference in Mechanical Engineering Research and Postgraduate Students 26-27 MAY 2010.

[6] SAE International BAJA SAE Rules 2014 http://www.sae.org/students/2014_baja_rule s_8-2103.pdf 2. Race car Vehicle Dynamics: Milliken and Milliken 3. Car suspension and Handling: Geoffrey Donald Bastow 4. Automobile Engineering, Kirpal Singh 5. Engineering Data Book 6. Strength of Materials, Stephen Timoshenko. 